OPEN ACCESS

Edited by:

Alfredo Addeo,

Geneva University Hospitals

(HUG), Switzerland

Reviewed by:

Marzia Del Re,

University of Pisa, Italy

Timothee Olivier,

Geneva University Hospitals

(HUG), Switzerland

*Correspondence:

Hicham Mansour

h.mansour@ump.ac.ma;

mansour.hicham@gmail.com

Specialty section:

This article was submitted to

Thoracic Oncology,

a section of the journal

Frontiers in Oncology

Received: 23 March 2020

Accepted: 15 June 2020

Published: 05 August 2020

Citation:

Mansour H, Ouhaijou A, Bajic VB and

Incitti R (2020) Next-Generation

Sequencing at High Sequencing Depth as a Tool to Study the Evolution

of Metastasis Driven by Genetic

Change Events of Lung Squamous

Cell Carcinoma.

Front. Oncol. 10:1215.

doi: 10.3389/fonc.2020.01215

\section{Next-Generation Sequencing at High Sequencing Depth as a Tool to Study the Evolution of Metastasis Driven by Genetic Change Events of Lung Squamous Cell Carcinoma}

\author{
Hicham Mansour $^{1 *}$, Abdelhak Ouhajjou ${ }^{2}$, Vladimir B. Bajic ${ }^{3}$ and Roberto Incitti ${ }^{3}$ \\ 1 GES-LCM2E, FPN, Mohamed First University, Oujda, Morocco, ${ }^{2}$ Al-Azhar Oncology Center, Rabat, Morocco, ${ }^{3}$ CBRC, King \\ Abdullah University of Science and Technology (KAUST), Thuwal, Saudi Arabia
}

Background: The aim of this study is to report tumoral genetic mutations observed at high sequencing depth in a lung squamous cell carcinoma (SqCC) sample. We describe the findings and differences in genetic mutations that were studied by deep next-generation sequencing methods on the primary tumor and liver metastasis samples. In this report, we also discuss how these differences may be involved in determining the tumor progression leading to the metastasis stage.

Methods: We followed one lung SqCC patient who underwent FDG-PET scan imaging, before and after three months of treatment. We sequenced 26 well-known cancer-related genes, at an average of $\sim 6,000 \times$ sequencing coverage, in two spatially distinct regions, one from a primary lung tumor metastasis and the other from a distal liver metastasis, which was present before the treatment.

Results: A total of 3,922,196 read pairs were obtained across all two samples' sequenced locations. Merged mapped reads showed several variants, from which we selected 36 with high confidence call. While we found $83 \%$ of genetic concordance between the distal metastasis and primary tumor, six variants presented substantial discordance. In the liver metastasis sample, we observed three de novo genetic changes, two on the FGFR3 gene and one on the CDKN2A gene, and the frequency of one variant found on the FGFR2 gene has been increased. Two genetic variants in the HRAS gene, which were present initially in the primary tumor, have been completely lost in the liver tumor. The discordant variants have coding consequences as follows: FGFR3 (c.746C>G, p. Ser249Cys), CDKN2A (c.47_50delTGGC, p. Leu16Profs*9), and HRAS (c.182A>C, p. Gln61Pro). The pathogenicity prediction scores for the acquired variants, assessed using several databases, reported these variants as pathogenic, with a gain of function for FGFR3 and a loss of function for CDKN2A. The patient follow-up using imaging with 18F-FDG PET/CT before and after four cycles of treatment shows discordant tumor progression in metastatic liver compared to primary lung tumor. 
Conclusions: Our results report the occurrence of several genetic changes between primary tumor and distant liver metastasis in lung SqCC, among which non-silent mutations may be associated with tumor evolution during metastasis.

Keywords: lung cancer, NGS, FGFR, HRAS, CDKN2, tumor evolution

\section{BACKGROUND}

Lung cancer is the most common type of cancer and the leading cause of cancer-related deaths for both men and women in the world $(1,2)$. There are two major types of lung cancer, namely, non-small cell lung carcinoma (NSCLC) and small cell lung carcinoma. NSCLC can be further divided into three main subtypes: large cell lung carcinoma, squamous cell carcinoma, and adenocarcinoma. Lung SqCC accounts for almost $30 \%$ of NSCLC, while there are also several other types that occur less frequently $(3,4)$. Significant progress on cancer research was made possible by the development of next-generation sequencing (NGS) (5-8) and, subsequently, by extensive research efforts on several tumors and patients (9-11). The body of knowledge on cancer genetics has increased sizably, and, depending on cancer type, researchers have identified several target genes that could be involved in cancer development, to improve patient care and to enhance the survival rate (12-16). Clinical research efforts to provide a comprehensive landscape of molecular alterations of lung SqCC have successfully characterized this cancer at the genetic level $(9,17)$. Researchers from the Cancer Genome Atlas (TCGA) identified several genes that are aberrantly expressed and/or mutated in lung SqCC patients. Major alterations have been described in TP53, PI3K, CDKN2A, SOX2, CCND1, ERBB, MET, DDR2, and FGFR1 (18-29). In particular, identification of genetic amplification in FGFR1, mutations in ERBB family, and abnormalities in PI3K have raised considerable interest in view of applications to the therapeutic management of SqCC patients. Furthermore, in multiple clinical trials, some of these genetic alterations have been targeted and have shown promising progress in treating SqCC (LUME-Lung 1, LUX-Lung 8, BASALT-1 phase II, FLEX, SQUIRE, and others) (30-36). Unfortunately, the observed benefits have been sporadic and heterogeneous, leading to some disappointment (18). Recently, the understanding of the complex interactions between the immune system and cancer has led to the development of immune checkpoint inhibitors (ICI). The combination of chemotherapy and ICI, in the firstline treatment of metastatic SqCC, has shown higher overall survival (OS) and progression-free survival (PFS) than the use of chemotherapy alone (37-39). Unfortunately, several studies have shown that only a subset of patients could benefit from this therapeutic protocol, such as patients with wild-type EGFR or negative ALK rearrangements $(40,41)$. Moreover, in different PD-L1 expression subgroups, the benefit in OS and PFS is mixed (42).

Because of the heterogeneity of the tumor at genetic and molecular levels, treatment of SqCC continues to remain a challenge. There may be several reasons due to the existence of different genomic patterns in SqCC, and the genetic targets that have been found so far are probably not driver mutations and are therefore currently unactionable or "undruggable" mutations for this disease (18). Several available treatments do not exhibit efficacy in metastasis lung SqCC, leaving these patients with fewer and inefficient treatment options, a situation, which could explain the poor survival and the frequent relapse of these patients. Thus, the identification of potential targets in SqCC has become urgent and essential.

The spread of cancer cells from primary tumors to other vital organs is often associated with poor prognosis $(43,44)$. Given its potential impact on patient care, understanding the genetic differences between primary tumors and distant metastasis is critical and will have value in terms of treatment strategies. A better understanding of the dynamic of the molecular evolutionary process of the lung SqCC will provide greater insight toward the development of a suitable therapeutic guide $(45,46)$. In this study, we used tumor genetic data generated by NGS, to investigate if tumor heterogeneity occurs between primary tumor and liver metastatic sites in lung SqCC and to detect non-silent mutations, which may be associated with tumor evolution, and its evolutionary trajectory from primary tumor through relapsed disease.

\section{CASE PRESENTATION}

\section{Patient}

A 72-year-old African man was presented to our clinic in 2016, with a worsening dyspnea and chronic dry cough. Symptoms included fatigue and muscle atrophy, with pain in the chest and right rib cage. He suffered from cervical disc herniation and frequent reversible heart palpitation. The patient was a former smoker, consuming one pack per day for 16 years until he was 32 years old. In the previous two years, he had a significant weight loss of $20 \mathrm{~kg}$. The physical exam shows a decrease in vesicular murmur and vocal vibrations of the right lung with no evidence of lymphadenopathy. The abdominal echography shows hepatomegaly. A suspicious mass for pulmonary neoplasm was found on chest X-ray. A CT scan detected a liver mass of $6 \mathrm{~cm}$ in the segment IV. Two biopsies were performed, one in the mediastinum (sample 69380-1-S7) and another in the liver (sample 69381-2-S8), and pathological examination confirmed the presence of squamous cell lung carcinoma, NSCLC at stage IVB (T4N2aM1c). The patient underwent FDG-PET scan imaging as part of the routine diagnosis and staging, before starting the chemotherapy treatment. Two hypermetabolic 
masses were localized; the maximum standardized uptake value (SUVmax) was assessed for these masses. On 26th December 2016, he received the first cure with paclitaxelcarboplatin. On 31st December 2016, he was admitted in the hospital for dysenteric syndrome and hemorrhoids and was treated with loperamide, diosmectite, metronidazole, lidocaine hydrochloride, diosmin, and hesperidin. On 19th January and 9th February 2017, he received the second and third cure with paclitaxel and carboplatin, respectively. Subsequently, on 13th March 2017, a second FDG-PET scan was performed to assess tumor progression. On 16th March 2017, he received the fourth cure with paclitaxel-carboplatin. A complete blood count was performed before each treatment, which was normal. A poor tolerance to chemotherapy was observed, as characterized with the following: nausea, diarrhea, loss of appetite, overall condition deterioration, and neurological disorders. Thus, the combination of immune checkpoint inhibitor and chemotherapy was not recommended and PD-L1 expression level was not investigated. Tyrosine kinase inhibitors (TKIs) were suggested instead. The patient refused to continue with the treatment. Nevertheless, he gave his consent to conducting the genetic analysis and for the publication of the present case report.

\section{Sample Processing}

Microdissections from formalin-fixed paraffin-embedded (FFPE) specimens of these biopsies were performed, ensuring that a tissue area that contained a high percentage of tumor cells would be used for DNA extraction. gDNA was extracted using the QIAamp DNA Micro Kit (Qiagen), then the sequencing library was prepared and was submitted for sequencing on MiSeq (Illumina). We used an existing commercial gene panel test, the somatic Tumor Hotspot MASTR ${ }^{\mathrm{TM}}$ Plus with MID for sequencing library preparation (Multiplicom/Agilent). The assay developed for the identification of SNVs in 26 frequently mutated genes in solid tumors. This NGS assay was designed with the input from the French INCA centers (https://www.agilent.com/cs/library/datasheets/public/TUMOR \%20HOTSPOT\%20MASTR\%20PLUS\%205991-8378ENN.pdf). This panel targets the following 26 genes: ALK, PTEN, PIK3R1, EGFR, NRAS, STK11, ERBB4, ERBB2, AKT1, HIST1H3B, HRAS, PDGFRA, MAP2K1, CDKN2A, IDH1, H3F3A, IDH2, BRAF, PIK3CA, KIT, CTNNB1, KRAS, FGFR3, MET, FGFR2, and DDR2.

\section{Data Analysis}

We filtered the sequencing reads with a Q-score higher than 20 , merged the read pairs, and mapped them on the human genome reference GRCh37/hg19. We filtered out duplicate reads, resulting from clonal amplification of the same fragments during library construction, and the regions which showed mapping coverage of less than $50 \times$. We performed variant detection analysis for SNPs, insertions, and deletions using Sophia Genetics DDM platform v4.5. Only high-confidence variants were retained according to the following criteria: (1) occurring in a high-complexity region, (2) having high, or higher than expected, variant fraction (germline), (3) having a sequencing coverage $>50 \times$, (4) having $>50$ supporting reads, and (5) being aligned inside the target region. The copy number variation analysis was not investigated in the present study. Variants were ranked by concordance and discordance between the two samples, and annotation information such as coding consequences was taken forward for further analysis. For each variant, we also reported the variant frequency, defined as variation percent $=($ altNum/depth $) \times 100$, where "altNum" is the number of reads containing the variant of interest and "depth" is the sum of number of reads containing the variant and the reads covering the reference allele. To obtain pathogenicity prediction scores, the target variants were submitted to the following databases made available by the Sophia Genetics DDM platform: COSMIC, Clinvar, OncoKB, cBioPortal, Varsome, Mutationassessor, Provean, FATHMM, MutationTaster, FATHMM-MK, MetaSVM, MetalR, LRT, SIFT, dbSNP, ClinVitae, and Provean. The protein structures encoded by each mutated gene were submitted to the SMART tool (47), to describe the biological consequence of each mutation on the functional domain of the protein.

\section{RESULTS}

\section{Image Analysis With 18F-FDG PET/CT}

Two intense hypermetabolic masses were revealed by $18 \mathrm{~F}$ FDG PET/CT (Figure 1). They were found into the anterosuperior mediastinum and in the hepatic parenchyma. The SUVmax of the hypermetabolic masses was assessed before and after treatment. Before treatment, the SUVmax of the mediastinal mass was 7.3 and the hepatic mass was 9.4. After treatment, the mediastinal mass increased to 10.4 , while the liver hypermetabolic mass decreased significantly to 3.9. While the primary lesion showed continuous tumor progression, the liver lesion showed significant improvement.

\section{Sequencing Data Statistics}

The mediastinal and liver samples were sequenced, resulting in $1,661,981$ and 2,260,216 read pairs, respectively. Ninety percent of the total reads were above Q35, which is considered as a high threshold for read quality. The mapping of merged reads on the targets genes resulted in 1,628,242 reads (>97\%) successfully mapped for the mediastinal sample and 2,134,774 reads (>94\%) for the liver sample. In the present experiments, we achieved a high sequencing coverage of the target region. The mean target coverage in this study was around $6,000 \times$. The target region coverage distribution for both samples is shown in Figure 2C and Figure S1.

\section{Finding Common and Discordant Variants}

In the lung sample, 12 out of the 26 genes sequenced presented with genetic changes, with 33 variants retained for highconfidence call and 28 discarded. In the liver sample, 13 genes presented with genetic changes, with 36 variants found and 24 discarded. The comparison between the distal metastasis and the primary tumor shows 30 shared variants and 6 distinct variants. When analyzing the variant frequency of the shared variants and using primary tumor data as baseline, we can observe several clusters (Figure 2). 


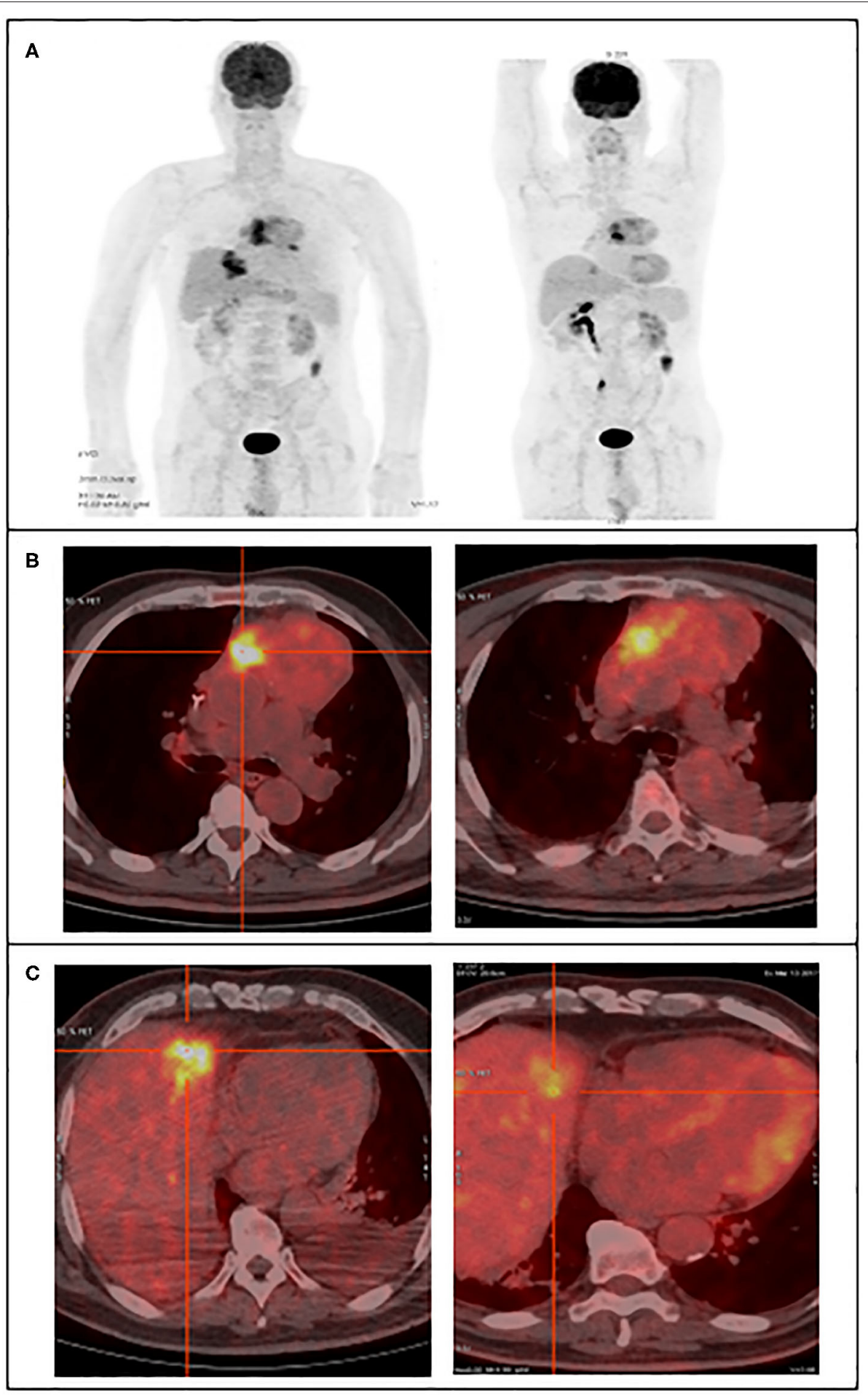

FIGURE 1 | 18F-FDG PET/CT images reveal the large hypermetabolic masses, in the superior mediastinum and the hepatic parenchyma, left figures before treatment and right figures post-treatment. (A) Maximum intensity projection image, (B) transaxial fusion image showing the mediastinal tumor, (C) transaxial fusion image showing the liver tumor.

We found 30 variants shared between primary tumor and metastasis, distributed across 12 genes, and having a similar frequency between the two samples. Among these variants, 6 have genetic changes in the coding sequence of the following genes: ALK (3 variants), CDKN2A (2), and PIK3CA (1) (Figures 2, 3). 


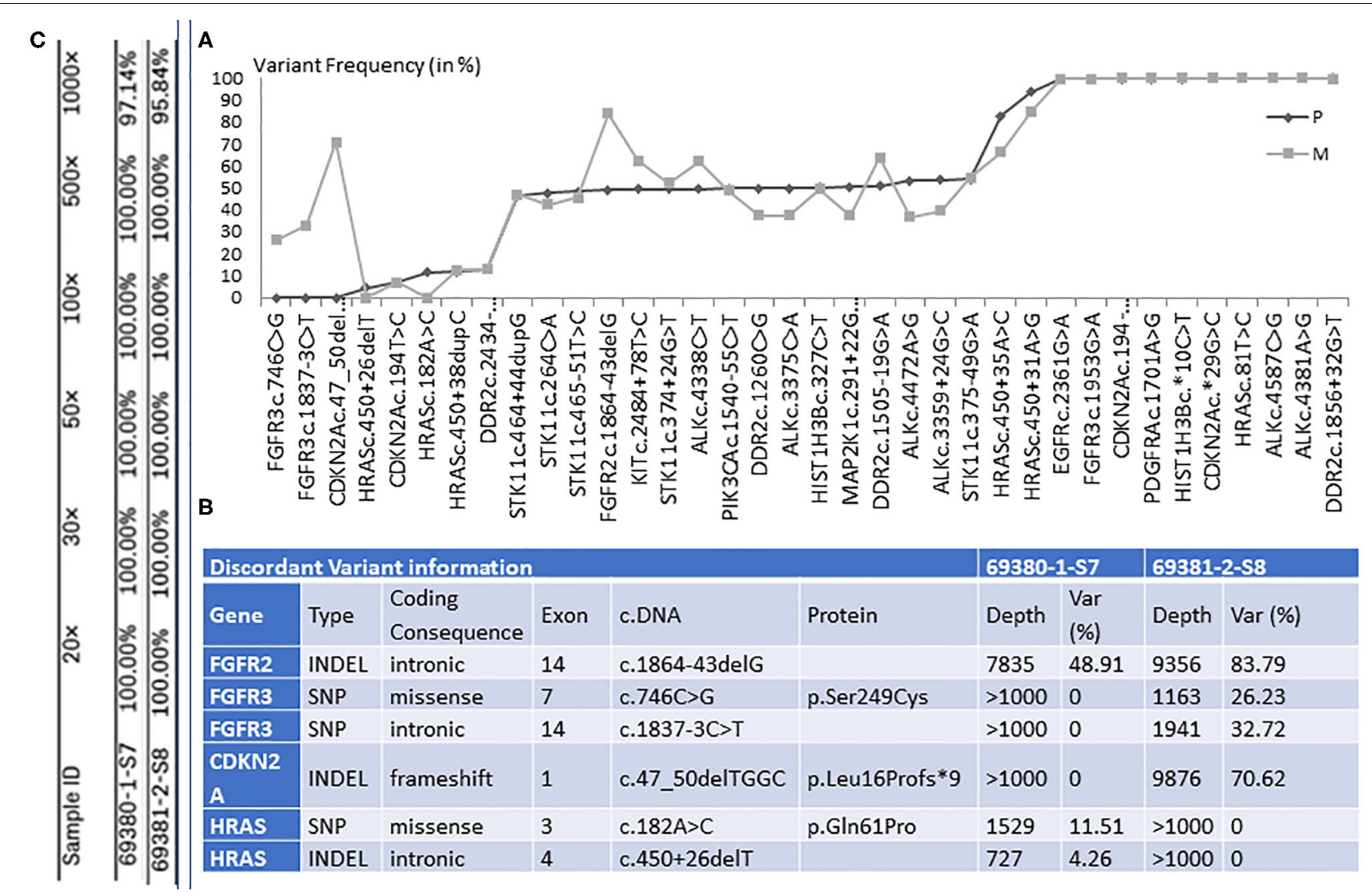

FIGURE 2 | (A) The graphical representation of retained genetic variants and their frequency (var \%), in primary tumor (P) and distal metastasis (M). (B) The illustration of the six discordant variants, among which three with coding consequences. Table (C) shows the percentage of target regions with certain coverage for both samples.

We found six discordant variants, covering the following genes: FGFR2, FGFR3, CDKN2A, and HRAS. Among these variants, only three variants had coding consequences as follows: a missense c.182A $>\mathrm{C}$ (p.Gln61Pro) on the HRAS gene, a frameshift c.47_50delTGGC (p.Leu16Profs*9) on CDKN2A gene, and a missense c.746C $>\mathrm{G}$ (p.Ser249Cys) on the FGFR3 gene. The HRAS (p.Gln61Pro) variant is found in primary lung tumor with a frequency of $11.5 \%$ and completely absent in the liver metastasis. The CDKN2A (p.Leu16Profs*9) and FGFR3 (p.Ser249Cys) variants are present only in the liver metastasis at a frequency of 70.6 and 26.2\%, respectively (Figures 2, 3). The full list of variants is publicly available in Clinvar database (www.ncbi.nlm.nih.gov/clinvar/), under the accession numbers SCV001250917 to SCV001250974.

\section{Pathogenicity Prediction}

The pathogenicity prediction of the three discordant variants with coding consequences shows the following:

1/ The FGFR3 missense c.746C >G (p.Ser249Cys) is located between two functional domains, in a relatively conserved position, and is predicted to have a damaging, disease-causing, and high effect on protein function (Figure 4). This is the most frequently observed FGFR3 variant found in cancer studies and is reported as oncogenic with a gain of function. The pathogenicity prediction of this genetic variation confirmed its association with several cancers, including tumors of the lung and other tumors: the urinary bladder, the urinary tract, the head, the neck, the upper aero-digestive tract, the thymus, and the cervix. This genetic variant is reported as a recurrent hotspot mutation in these tumors. It could be either germline or somatic and is classified as a pathogenic allele.

2/ The CDKN2A, frameshift c.47_50delTGGC (p. Leu16Profs*9), is located at a relatively conserved position and predicted to have a high effect on protein function (Figure 4). The variant has a damaging, disease-causing effect on protein function and has been shown to be involved in pancreas carcinoma and thymoma. It has been reported as both somatic and germline mutation and has been classified as a pathogenic allele, associated with hereditary cancer predisposing syndrome.

3/ The variant HRAS, missense on c.182A >C (p. Gln61Pro), is located in the Ras domain and has been found to be expressed in the upper aero-digestive tract and in thyroid cancers (Figure 4). This variant is reported in both germline and somatic and is classified as a pathogenic allele. It has a 


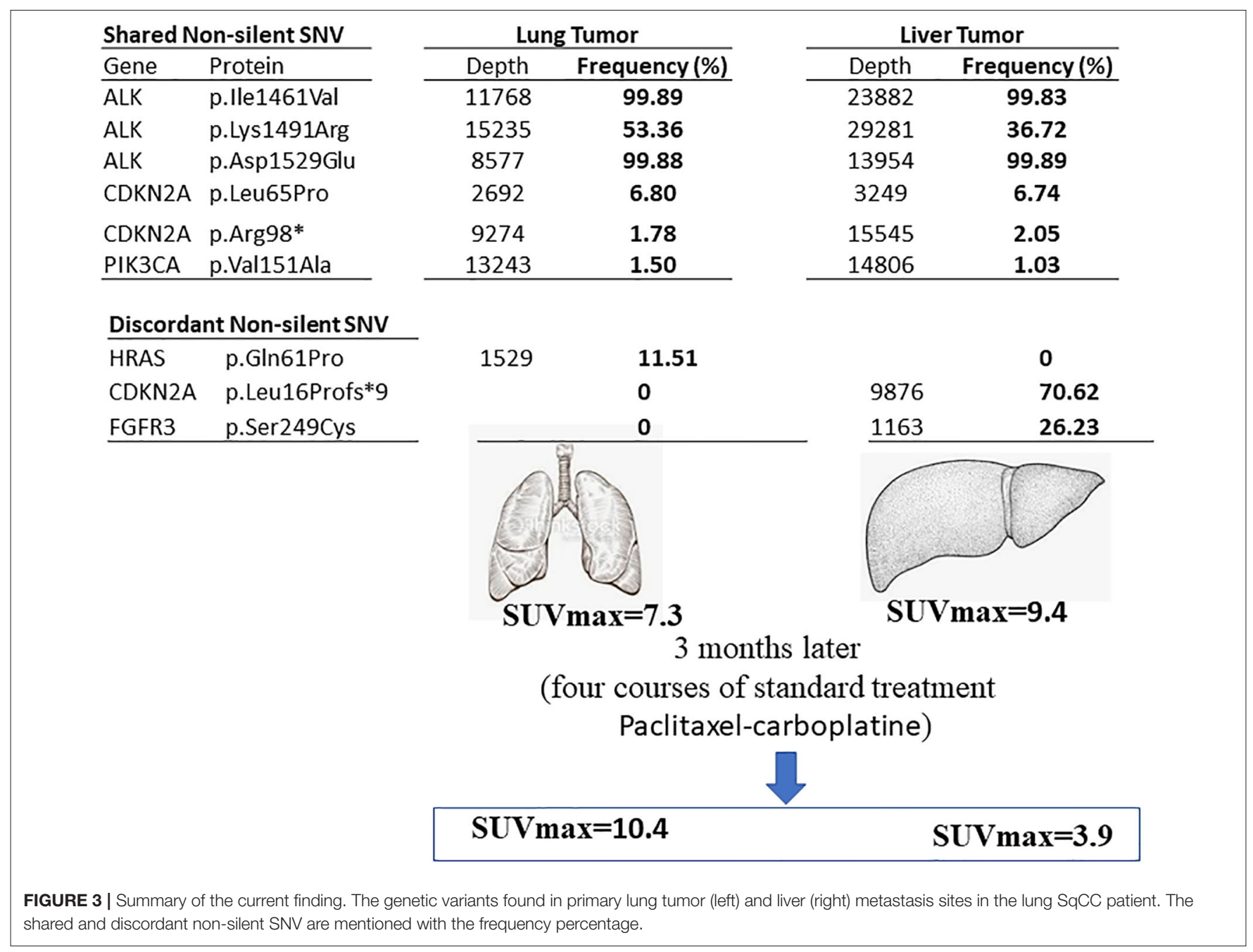

damaging, disease-causing effect on protein function. We did not find relevant hits for this particular variant in OncoKB, cBioPortal, or My Cancer Genome.

\section{DISCUSSION}

Extensive clinical research efforts and advancements that have been made since the human genome was sequenced have greatly increased our knowledge on cancer genetics. Several genetic alterations have been shown to initiate and sustain specific deregulated cellular signaling pathways involved in various tumors. In the present study, using one lung SqCC patient, we screened 26 genes that are well known as potential driver cancer genes, using next-generation sequencing. Though there are many known gene panels containing more genes, we limited our analysis to the current panel, to achieve high sequencing coverage. The panel that we used has been previously validated as clinically relevant in a wide variety of solid tumors. Moreover, this multiple-gene panel focuses on actionable hotspot mutations, which have therapeutic as well prognostic values for different solid tumors, enabling a personalized medicine approach.

We performed in-depth investigation looking for SNVs and indels on the target genes. For each gene, both coding and noncoding regions were covered. We found that $83 \%$ of the retained variants are being shared by distant metastasis and primary tumor. Moreover, for each of these concordant variants, the variation frequency shows a strong concordance between the two tumor sites, with the presence of distinguished clusters, mainly containing both low- and high-frequency variants. Among the shared variants, we detected six non-silent SNVs, among which three variants at high frequency (36-99\%) in the ALK gene, and at low frequency (1-6\%): two in PIK3CA and one in CDKN2A. The variants CDKN2A (p.Leu65Pro) and ALK (p.Ile1461Val) were found in another patient also with lung SqCC, with the same variant frequency (data not shown). Somatic alterations of ALK gene are reported relatively rarely in squamous cell carcinoma (SqCC) (48), but, on the other hand, CDKN2A alterations are common and have been reported in 


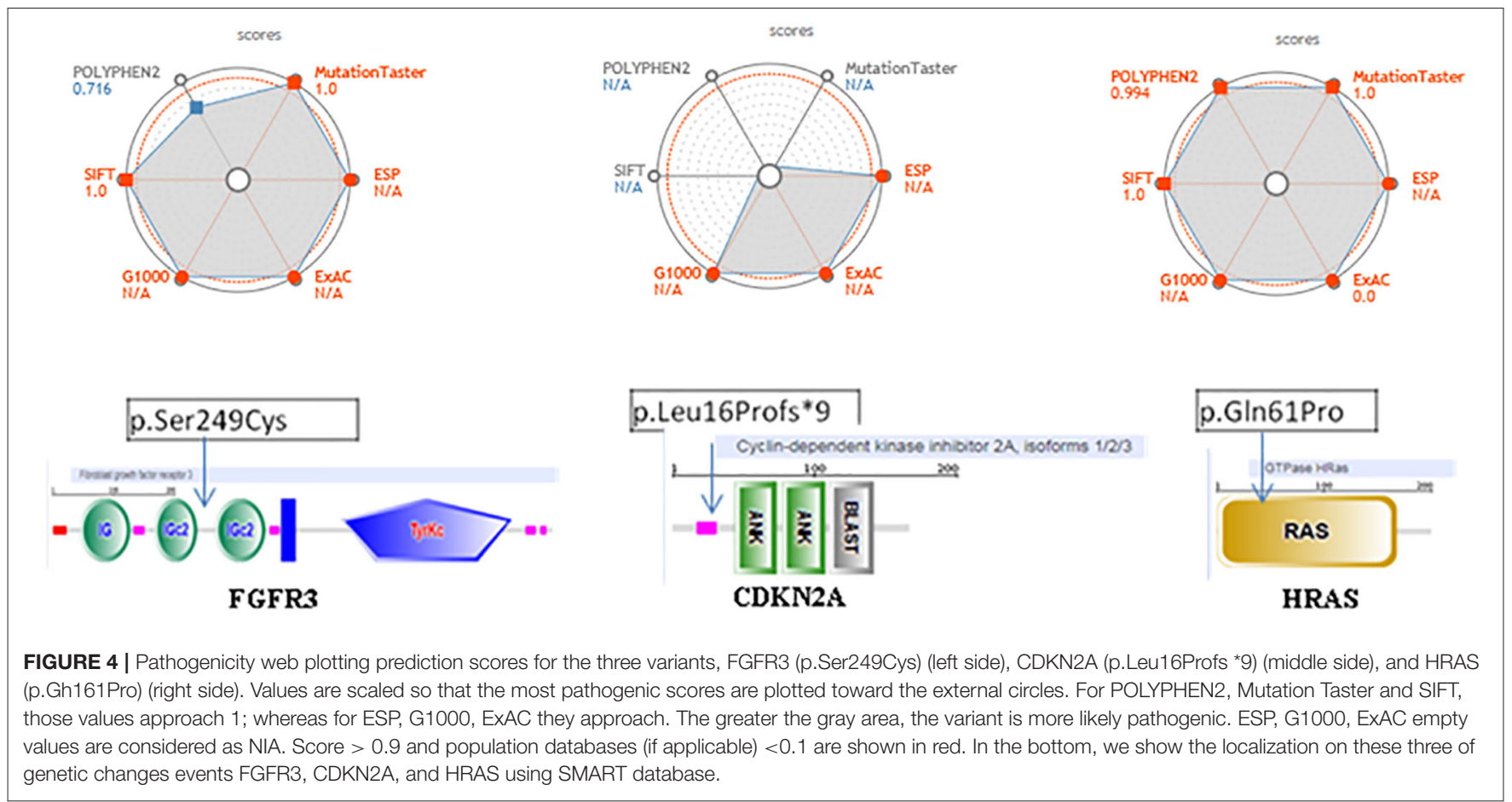

recent genomic studies of lung SqCC (49). Interestingly, in one recent study, which included three patients with lung SqCC, the rate of concordance in somatic mutations between local metastasis in distinct lung lymph nodes and primary lung cancer varies from 15 to $82 \%$, and some nodes have highly similar somatic mutation profiles (45). In another study which analyzed two lung SqCC patients, the rate of concordance in somatic mutations between distinct lung lymph nodes and primary lung cancer was identified, with $37 \%$ for one patient and $96 \%$ for the other (46). Spatial tumor heterogeneity in lung SqCC patients occurs frequently and differs significantly from one patient to another. Typically, in routine clinical practice a single biopsy is performed, to define a driver mutation and thus to suggest the optimal treatment. The occurrence of tumor heterogeneity, which was observed in the current and previous works, could make the use of a single solid biopsy ineffective. Liquid biopsy could be an actionable option to perform a serial monitoring of the tumor burden and get a comprehensive view on the genetic changes of the tumor cell diversification that could take place during distant metastasis.

In the present report, six genetic variations affecting four genes (FGFR2, FGFR3, CDKN2A, and HRAS) are shown to have a substantial discordance between the liver metastatic site and the primary tumor of the lung SqCC. Four genetic changes were gained on the genes FGFR2, FGFR3, and CDKN2A, and two genetic variants were lost on HRAS, in the metastatic tumor. Interestingly, after four cycles of treatment, a strong signal of resolution was detected at the liver metastasis through a significant sink over $60 \%$ of the tumor size. In contrast, the primary tumor showed a progression. These mixed outcomes after treatment may be related to the intra-tumor genetic heterogeneity of this lung SqCC patient. The discordant variants found in the current report have low frequency; it is possible that primary tumor cells with high-frequency genetic variants resist during the metastasis events, and a new clone harboring new low-frequency genetic variants emerges. These low-frequency variations might make metastatic tumor cells respond differently to chemotherapy treatment than the primary tumors.

Three of the discordant genetic changes have coding consequences, as follows: FGFR3 (p. Ser249Cys), CDKN2A (p. Leu16Profs*9), and HRAS (p. Gln61Pro). None of these nonsilent mutations were found in the study of De Bruin or Um $(45,46)$. One explanation could be that the high sequencingcoverage depths, which were performed in this study, have resulted in identification of non-silent mutations. The advantage of performing whole-genome or exome sequencing would allow comprehensive understanding of the functional alterations found in lung cancer, but this approach could neglect rare or lowfrequency variants. Sufficient base coverage of the targeted region, defined as the number of reads covering a base, is an important prerequisite for reliable variant detection from NGS data. In the present study, the cost of high sequencing coverage limited the number of assayed genes compared to the Bruin and Um studies. Nevertheless, the mean target coverage in these studies was $\sim 100 \times$, while in our study all the target regions are covered in average $\sim 6,000 \times$ and at least $500 \times$ (Figure S1).

FGFR3 (fibroblast growth factor receptor 3) is a member of the fibroblast growth factor receptor family (FGFR). Alterations 
in the FGFR kinase family are common in lung SqCC and comprise the most frequently altered tyrosine kinase family $(3,50,51)$. The mechanism of activation of FGFR3 kinase was previously established for the observed variant FGFR3 (p. Ser249Cys). The presence of the mutant cysteine amino acid instead of serine residue allows, via disulfide bound, the receptor to dimerize abnormally, resulting in ligand-independent constitutive activation in the absence of the ligand and to structural perturbation of the dimers (49). Using cell culture and tumor xenograft models, researchers described the oncogenic nature of this observed FGFR3 mutation and showed that it could drive cellular transformation (49). Cancer cell lines harboring this variant have demonstrated sensitivity to the inhibition by FGFR inhibitors and that the cell transformation can be reversed (17, 52-55). The variant CDKN2A (p. Leu16Profs*9) is reported in somatic pancreas carcinoma and thymoma, also as a germline variant associated with hereditary cancer-predisposing syndrome. According to several databases, this variant is located at a relatively conserved position and is predicted to have a strong effect on protein function; the variant has a damaging, disease-causing effect on protein function $(56,57)$. The specific variant HRAS (p. Gln61Pro) is located at a medium-conserved position, reported as both germline and somatic mutation. This variant has been reported as having a medium effect on protein function and classified as a pathogenic allele. However, in the study of functional consequences at a specific mutation hotspot on the HRAS gene, the researchers show that Q61P and Q61E variants did not show increased transforming ability relative to HRAS wild-type (wt) mouse (58). Until recently, specialists just cataloged cancers as either wt or mutant for RAS (59), to predict resistance to targeted therapies, and no treatment antiRAS therapies exist yet in the clinic (58). In Um's study, one genetic variant of HRAS (Q61R) was found, at low frequency, in only one SqCC patient, in the primary tumor as well as in one lung lymph node, but not in the other lymph node analyzed (45).

The metastatic event in lung cancer involves dissemination of cancer cells to anatomically distant organ sites, such as the liver and brain, and their subsequent adaptation to distant tissue microenvironments. Metastasis encompasses several biological processes, which consists of a complex series of well-organized steps: tumor cells exit their primary sites of growth by local invasion and intravasation, translocate systemically, survive in the circulation, arrest, affect the distant organ by extravasation, and adapt to survive and thrive in the initially healthy microenvironments of distant tissues $(43,44)$. Metastasis cascades are controlled by specific classes of genes, among which researchers have described metastasis initiation, progression, and virulence genes $(60,61)$. The relevant variants on FGFR3 and CDKN2A genes found in the present study are probably part of these virulence genes, which potentially act to promote metastasis, provide a selective advantage in secondary sites over the primary tumor, and enable the colonization of distant organs. Our case report opens the door to expand the number of tumor pairs to be examined. The analysis of more samples will better assess the clinical impact of the detected variants. It could also reveal more non-silent variants and unravel how these variants are involved in cancer evolution mechanisms and in the clinical outcome of SqCC patients. To this respect, TracerX is a large project aiming at analyzing tumoral heterogeneity in 850 NSCLC patients from several subtypes (http://www.cruklungcentre.org/ Research/TRACERx). One of the challenges faced in oncology is that the genotype does not correlate with the phenotype, for some patients. Consequently, this could result in significant interpatient variability of drugs response, even for patients harboring the same molecular alteration. The molecular analysis of this report is restricted to SNVs and indels. For future study, it is important to expand the genetic analysis to other alterations and to assess their correlation with gene expression and protein levels.

In this work, we shed light on cell molecular changes happening between the primary and metastasis tumors to the liver of lung SqCC, by targeting, at high sequencing depth, 26 well-known cancer-related genes. Our results show in lung SqCC the following: (1) the occurrence of genetic changes between primary tumor and distant metastasis to the liver, (2) the gain of function in the FGFR3 and probably the FGFR2 genes, and (3) the loss of function of CDKN2A. It would be interesting, for a more in-depth study, to investigate the prevalence of these variants in metastatic lung SqCC, in order to reveal the exact role of these variants in tumor evolution during each metastasis step and to assess the efficacy of treatments targeting these variants in the hope of opening opportunities for new efficient therapies for this disease.

\section{DATA AVAILABILITY STATEMENT}

The datasets presented in this study can be found in online repositories. The names of the repository/ repositories and accession number(s) can be found below: https://www.ncbi.nlm.nih.gov/clinvar?term=SUB7198155\%5BSu bmitter\%20Batch\%5D.

\section{ETHICS STATEMENT}

Ethical review and approval was not required for the study on human participants in accordance with the local legislation and institutional requirements. The patients/participants provided their written informed consent to participate in this study.

\section{AUTHOR'S NOTE}

Our major finding, we determined the genetic changes which occur between primary tumor and distal metastasis site in Lung SqCC.

\section{AUTHOR CONTRIBUTIONS}

$\mathrm{HM}$ and $\mathrm{AO}$ designed and carried out the experiments. HM and $\mathrm{RI}$ analyzed the data. HM, VB, RI, and AO contributed to the interpretation of the results. HM wrote the paper with input from 
all authors. All authors discussed the results and commented on the manuscript.

\section{ACKNOWLEDGMENTS}

This paper is dedicated to Professor VB, in memoriam. We would like to thank Dr. Kumaran Mande for his scientific comments and Mr. Ismail Hadouch for the ${ }^{18}$ F-FDG PET/CT images and

\section{REFERENCES}

1. Howlader N, Noone AM, Krapcho M, Garshell J, Miller D, Altekruse SF, et al. SEER cancer statistics review, 1975-2011. Bethesda, MD: National Cancer Institute. Available online at: http://seer.cancer.gov/csr/1975_2011/ (based on November 2013) SEER data submission, posted to the SEER website (2014).

2. Spira A, Halmos B, Powell CA. Update in lung cancer 2015. Am J Respir Crit Care Med. (2016) 194:661-71. doi: 10.1164/rccm.201604-0898UP

3. Liao RG, Watanabe H, Meyerson M, Hammerman PS. Targeted therapy for squamous cell lung cancer. Lung Cancer Manag. (2012) 1:293300. doi: $10.2217 / \mathrm{lmt} .12 .40$

4. Lemjabbar-Alaoui H, Hassan OU, Yang YW, Buchanan P. Lung cancer: Biology and treatment options. Biochim Biophys Acta. (2015) 1856:189210. doi: 10.1016/j.bbcan.2015.08.002

5. Braslavsky I, Hebert B, Kartalov E, Quake SR. Sequence information can be obtained from single DNA molecules. Proc Natl Acad Sci U S A. (2003) 100:3960-4. doi: 10.1073/pnas.0230489100

6. International Human Genome Sequencing Consortium. Initial sequencing and analysis of the human genome. Nature. (2001) 409:860-921. doi: 10.1038/35057062

7. Margulies M, Egholm M, Altman WE, Attiya S, Bader JS, Bemben LA, et al. Genome sequencing in microfabricated high-density picolitre reactors. Nature. (2005) 437:376-80. doi: 10.1038/nature03959

8. Voelkerding KV, Dames SA, Durtschi JD. Next-generation sequencing: from basic research to diagnostics. Clin Chem. (2009) 55:641-58. doi: 10.1373/clinchem.2008.112789

9. Cancer Genome Atlas Research Network. Comprehensive genomic characterization of squamous cell lung cancers. Nature. (2012) 489:519-25. doi: 10.1038/nature11404

10. Sivakumar S, Lucas FAS, McDowell TL, Lang W, Xu L, Fujimoto J, et al. Genomic landscape of atypical adenomatous hyperplasia reveals divergent modes to lung adenocarcinoma. Cancer Res. (2017) 77:611930. doi: 10.1158/0008-5472.CAN-17-1605

11. Shull AY, Noonepalle SK, Lee EJ, Choi JH, Shi H. Sequencing the cancer methylome. Methods Mol Biol. (2015) 1238:62751. doi: 10.1007/978-1-4939-1804-1_33

12. Baron JA. Screening for cancer with molecular markers: progress comes with potential problems. Nat Rev Cancer. (2012) 12:368-71. doi: 10.1038/nrc3260

13. Verma M. Personalized medicine and cancer. J Pers Med. (2012) 2:114. doi: 10.3390/jpm2010001

14. Mansour H. Cell-free nucleic acids as noninvasive biomarkers for colorectal cancer detection. Front Genet. (2014) 5:182. doi: 10.3389/fgene.2014.00182

15. Schwaederle M, Chattopadhyay R, Kato S, Fanta PT, Banks KC, Choi IS, et al. Genomic alterations in circulating tumor DNA from diverse cancer patients identified by next-generation sequencing. Cancer Res. (2017) 77:5419-427. doi: 10.1158/0008-5472.CAN-17-0885

16. $\mathrm{Yu} \mathrm{T}, \mathrm{Zhao} \mathrm{Y}, \mathrm{Hu} \mathrm{Z}, \mathrm{Li}$, Chu $\mathrm{D}$, Zhang J, et al. MetaLnc9 facilitates lung cancer metastasis via a PGK1-activated AKT/mTOR pathway. Cancer Res. (2017) 77:5782-94. doi: 10.1158/0008-5472.CAN-1 7-0671

17. Wilkerson MD, Yin X, Hoadley KA, Liu Y, Hayward MC, Cabanski $\mathrm{CR}$, et al. Lung squamous cell carcinoma mRNA expression subtypes are reproducible, clinically important and correspond to normal cell types. Clin Cancer Res. (2010) 16:4864-75. doi: 10.1158/1078-0432.CCR-1 0-0199 we apologize to colleagues whose work we could not include due to space limitations.

\section{SUPPLEMENTARY MATERIAL}

The Supplementary Material for this article can be found online at: https://www.frontiersin.org/articles/10.3389/fonc. 2020.01215/full\#supplementary-material

18. Friedlaender A, Banna G, Malapelle U, Pisapia P, Addeo A. Next generation sequencing and genetic alterations in squamous cell lung carcinoma: where are we today? Front Oncol. (2019) 9:166. doi: 10.3389/fonc.2019.00166

19. Schwaederle M, Elkin SK, Tomson BN, Carter JL, Kurzrock R. Squamousness: Next-generation sequencing reveals shared molecular features across squamous tumor types. Cell Cycle. (2015) 14:2355-61. doi: 10.1080/15384101.2015.1053669

20. Paik PK, Shen R, Won H, Rekhtman N, Wang L, Sima CS, et al. Next-generation sequencing of stage IV squamous cell lung cancers reveals an association of $\mathrm{PI} 3 \mathrm{~K}$ aberrations and evidence of clonal heterogeneity in patients with brain metastases. Cancer Discov. (2015) 5:61021. doi: 10.1158/2159-8290.CD-14-1129

21. Dziadziuszko R, Wynes MW, Singh S, Asuncion BR, RangerMoore J, Konopa K, et al. Correlation between MET gene copy number by silver in situ hybridization and protein expression by immunohistochemistry in non-small cell lung cancer. J Thorac Oncol. (2012) 7:340-7. doi: 10.1097/JTO.0b013e318240ca0d

22. Hammerman PS, Sos ML, Ramos AH, Xu C, Dutt A, Zhou W, et al. Mutations in the DDR2 kinase gene identify a novel therapeutic target in squamous cell lung cancer. Cancer Discov. (2011) 1:7889. doi: 10.1158/2159-8274.CD-11-0005

23. Heist RS, Mino-Kenudson M, Sequist LV, Tammireddy S, Morrissey L, Christiani DC, et al. FGFR1 amplification in squamous cell carcinoma of the lung. J Thorac Oncol. (2012) 7:1775-80. doi: 10.1097/JTO.0b013e31826aed28

24. Monaco SE, Rodriguez EF, Mahaffey AL, Dacic S. FGFR1 Amplification in squamous cell carcinoma of the lung with correlation of primary and metastatic tumor status. Am J Clin Pathol. (2016) 145:55-61. doi: 10.1093/ajcp/aqv013

25. Flockerzi FA, Roggia C, Langer F, Holleczek B, Bohle RM. FGFR1 gene amplification in squamous cell carcinomas of the lung: a potential favorable prognostic marker for women and for patients with advanced cancer. Virchows Arch. (2018) 472:759-69. doi: 10.1007/s00428-017-2282-0

26. Campbell JD, Yau C, Bowlby R, Liu Y, Brennan K, Fan H, et al. Genomic, pathway network, and immunologic features distinguishing squamous carcinomas. Cell Rep. (2018) 23:194-212.e6. doi: 10.1016/j.celrep.2018.03.063

27. Guo Q, Sun Y, Yu S, Bai H, Zhao J, Zhuo M, et al. Programmed cell deathligand 1 (PD-L1) expression and fibroblast growth factor receptor 1 (FGFR1) amplification in stage III/IV lung squamous cell carcinoma (SQC). Thorac Cancer. (2017) 8:73-9. doi: 10.1111/1759-7714.12399

28. Lindquist KE, Karlsson A, Levéen P, Brunnström H, Reuterswärd C, Holm $\mathrm{K}$, et al. Clinical framework for next generation sequencing based analysis of treatment predictive mutations and multiplexed gene fusion detection in non-small cell lung cancer. Oncotarget. (2017) 8:34796810. doi: 10.18632 /oncotarget.16276

29. Okamoto T, Takada K, Sato S, Toyokawa G, Tagawa T, Shoji F, et al. Clinical and genetic implications of mutation burden in squamous cell carcinoma of the lung. Ann Surg Oncol. (2018) 25:1564-71. doi: 10.1245/s10434-018-6401-1

30. Goss GD, Felip E, Cobo M, Lu S, Syrigos K, Lee KH, et al. Association of ERBB mutations with clinical outcomes of afatinib- or erlotinibtreated patients with lung squamous Cell carcinoma: secondary analysis of the LUX-Lung 8 randomized clinical trial. JAMA Oncol. (2018) 4:118997. doi: 10.1001/jamaoncol.2018.0775

31. Kris MG, Camidge DR, Giaccone G, Hida T, Li BT, O’Connell J, et al. Targeting HER2 aberrations as actionable drivers in lung cancers: phase II trial of the pan-HER tyrosine kinase inhibitor dacomitinib in patients 
with HER2-mutant or amplified tumors. Ann Oncol. (2015) 26:14217. doi: $10.1093 / \mathrm{annonc} / \mathrm{mdv} 186$

32. Zhang J, Zhang L, Su X, Li M, Xie L, Malchers F, et al. Translating the therapeutic potential of AZD4547 in FGFR1-amplified non-small cell lung cancer through the use of patient-derived tumor xenograft models. Clin Cancer Res. (2012) 18:6658-67. doi: 10.1158/1078-0432.CCR-12-2694

33. Pirker R, Pereira JR, Szczesna A, von Pawel J, Krzakowski M, Ramlau R, et al. Cetuximab plus chemotherapy in patients with advanced non-small-cell lung cancer (FLEX): an open-label randomised phase III trial. FLEX Study Team. Lancet. (2009) 373:1525-31. doi: 10.1016/S0140-6736(09)60569-9

34. Thatcher N, Hirsch FR, Luft AV, Szczesna A, Ciuleanu TE, Dediu M, et al. Necitumumab plus gemcitabine and cisplatin versus gemcitabine and cisplatin alone as first-line therapy in patients with stage IV squamous nonsmall-cell lung cancer (SQUIRE): an open-label, randomised, controlled phase 3 trial. Lancet Oncol. (2015) 16:763-74. doi: 10.1016/S1470-2045(15)00021-2

35. Brahmer J, Reckamp KL, Baas P, Crinò L, Eberhardt WE, Poddubskaya E, et al. Nivolumab versus docetaxel in advanced squamous-cell non-small-cell lung cancer. N Engl J Med. (2015) 373:123-35. doi: 10.1056/NEJMoa1504627

36. Fehrenbacher L, Spira A, Ballinger M, Kowanetz M, Vansteenkiste J, Mazieres J, et al. Atezolizumab versus docetaxel for patients with previously treated non-small-cell lung cancer (POPLAR): a multicentre, open-label, phase 2 randomised controlled trial. Lancet. (2016) 387:183746. doi: 10.1016/S0140-6736(16)00587-0

37. Socinski MA, Jotte RM, Cappuzzo F, Orlandi F, Stroyakovskiy D, Nogami $\mathrm{N}$, et al. Atezolizumab for first-line treatment of metastatic nonsquamous NSCLC. N Engl J Med. (2018) 378:2288-301. doi: 10.1056/NEJMoa1716948

38. Jotte R, Cappuzzo F, Vynnychenko I, Stroyakovskiy D, Rodríguez-Abreu $D$, Hussein $M$, et al. Atezolizumab in combination with carboplatin and nab-paclitaxel in advanced squamous NSCLC (IMpower131): results from a randomized phase III trial. J Thorac Oncol. (2020) 15:1351-60. doi: 10.1016/j.jtho.2020.03.028

39. Paz-Ares L, Luft A, Vicente D, Tafreshi A, Gümüş M, Mazières J, et al. Pembrolizumab plus chemotherapy for squamous non-small-cell lung cancer. N Engl J Med. (2018) 379:2040-51. doi: 10.1056/NEJMoa1810865

40. Gainor JF, Shaw AT, Sequist LV, Fu X, Azzoli CG, Piotrowska Z, et al. EGFR mutations and ALK rearrangements are associated with low response rates to PD-1 pathway blockade in non-small cell lung cancer: a retrospective analysis. Clin Cancer Res. (2016) 22:4585-93. doi: 10.1158/1078-0432.CCR-15-3101

41. Lisberg A, Cummings A, Goldman JW, Bornazyan K, Reese N, Wang T, et al. A phase II study of pembrolizumab in EGFR-mutant, PD-L1+, tyrosine kinase inhibitor naïve patients with advanced NSCLC. J Thorac Oncol. (2018) 13:1138-1145. doi: 10.1016/j.jtho.2018.03.035

42. Addeo A, Banna GL, Metro G, Di Maio M. Chemotherapy in combination with immune checkpoint inhibitors for the first-line treatment of patients with advanced non-small cell lung cancer: a systematic review and literature-based meta-analysis. Front Oncol. (2019) 9:264. doi: 10.3389/fonc.2019.00264

43. Valastyan S, Weinberg RA. Tumor metastasis: molecular insights and evolving paradigms. Cell. (2011) 147:275-92. doi: 10.1016/j.cell.2011.09.024

44. Nathanson SD. Insights into the mechanisms of lymph node metastasis. Cancer. (2003) 98:413-23. doi: 10.1002/cncr.11464

45. Um SW, Joung JG, Lee H, Kim H, Kim KT, Park J, et al. Molecular evolution patterns in metastatic lymph nodes reflect the differential treatment response of advanced primary lung cancer. Cancer Res. (2016) 76:656876. doi: 10.1158/0008-5472.CAN-16-0873

46. de Bruin EC, McGranahan N, Mitter R, Salm M, Wedge DC, Yates L, et al. Spatial and temporal diversity in genomic instability processes defines lung cancer evolution. Science. (2014) 346:251-6. doi: 10.1126/science.1253462
47. Letunic I, Bork P. 20 years of the SMART protein domain annotation resource. Nucleic Acids Res. (2017) 46:493-96. doi: 10.1093/nar/gkx922

48. Mamesaya N, Nakashima K, Naito T, Nakajima T, Endo M, Takahashi T. ALK-rearranged lung squamous cell carcinoma responding to alectinib: a case report and review of the literature. BMC Cancer. (2017) 17:471. doi: 10.1186/s12885-017-3468-1

49. Liao RG, Jung J, Tchaicha J, Wilkerson MD, Sivachenko A, Beauchamp EM, et al. Inhibitor-sensitive FGFR2 and FGFR3 mutations in lung squamous cell carcinoma. Cancer Res. (2013) 73:5195-205. doi: 10.1158/0008-5472.CAN-12-3950

50. Plotnikov AN, Hubbard SR, Schlessinger J, Mohammadi M. Crystal structures of two FGF-FGFR complexes reveal the determinants of ligand-receptor specificity. Cell. (2000) 101:413-24. doi: 10.1016/S0092-8674(00)80851-X

51. FGFR3 Fibroblast Growth Factor Receptor 3 [Homo sapiens (Human)]. Gene ID: (2261). Available online at: https://www.ncbi.nlm.nih.gov/gene/2261 (Updated May, 16 2020).

52. Babina IS, Turner NC. Advances and challenges in targeting FGFR signalling in cancer. Nat Rev Cancer. (2017) 17:318-32. doi: 10.1038/nrc.2017.8

53. Greulich H, Pollock PM. Targeting mutant fibroblast growth factor receptors in cancer. Trends Mol Med. (2011) 17:28392. doi: 10.1016/j.molmed.2011.01.012

54. Turner N, Grose R. Fibroblast growth factor signalling: from development to cancer. Nat Rev Cancer. (2010) 10:116-29. doi: 10.1038/nrc2780

55. CDKN2A Cyclin Dependent Kinase Inhibitor 2A [Homo sapiens (Human)]. Gene ID: (1029). Available online at: https://www.ncbi.nlm.nih.gov/gene/ 1029?report=full_report (Updated May 17, 2020)

56. Goldstein AM. Familial melanoma, pancreatic cancer and germline CDKN2A mutations. Hum Mutat. (2004) 23:630. doi: 10.1002/humu.9247

57. ClinVar [VCV000142061.3]. National Center for Biotechnology Information. Available online at: https://www.ncbi.nlm.nih.gov/clinvar/variation/ VCV000142061.3 (Accessed May 19, 2020).

58. Hobbs GA, Der CJ, Rossman KL. RAS isoforms and mutations in cancer at a glance. J Cell Sci. (2016) 129:1287-92. doi: 10.1242/jcs.182873

59. HRAS HRas Proto-Oncogene, GTPase [Homo sapiens (Human)]. Gene ID: 3265. Available online at: https://www.ncbi.nlm.nih.gov/gene/3265 (Updated May 9, 2020).

60. Nguyen DX, Massagué J. Genetic determinants of cancer metastasis. Nat Rev Genet. (2007) 8:341-52. doi: 10.1038/nrg2101

61. Naxerova K, Reiter JG, Brachtel E, Lennerz JK, van de Wetering M, Rowan A, et al. Origins of lymphatic and distant metastases in human colorectal cancer. Science. (2017) 357:55-60. doi: 10.1126/science.aai8515

Conflict of Interest: HM and RI are cofounders of the diagnostic device company OncoDiag SAS, Paris, France. HM is also a genetic counselor at the Al-Azhar Oncology Center, Rabat, Morocco.

The remaining authors declare that the research was conducted in the absence of any commercial or financial relationships that could be construed as a potential conflict of interest.

Copyright (๑) 2020 Mansour, Ouhajjou, Bajic and Incitti. This is an open-access article distributed under the terms of the Creative Commons Attribution License (CC BY). The use, distribution or reproduction in other forums is permitted, provided the original author(s) and the copyright owner(s) are credited and that the original publication in this journal is cited, in accordance with accepted academic practice. No use, distribution or reproduction is permitted which does not comply with these terms. 